\author{
海洋维权的博弯问题

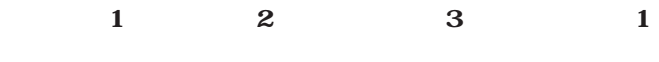 \\ (1. 中国科学院声学研究所, 北京 $100190 ; 2$. 北京联合大学商务学院, 北京 100025 ; \\ 3. 中国舰船研究设计中心, 武汉 430064)
}

\begin{abstract}
摘要: 本文以博弯论观点探讨海洋维权问题, 包括海洋维权在处理海洋争端中的作用和地位, 我国海洋维权博弯的性质、方 向及博亦要素。当前阶段, 推动海洋维权博亦微观化、事务性处理, 从维权斗争向联合管治方向发展符合我国的国家利益。 将作为海洋维权 “斗争前线” 的海上维权区分为 “争议海区进入” “渔船越界维权” “非合作渔业博弃模型” 3 类基本博交过程, 分别开展博峦分析, 据此研判我国海上维权力量建设的重点方向。研究表明: 提高海洋产业科技水平、发展维权技术手段可 以有效改变博弯收益, 从而实现有利的博弯均衡; 发展高技术干扰手段、水下防护技术、海洋广域监视技术和远海渔业开发 技术，是在重复博亦过程中获得优势地位的重要保障。
\end{abstract}

关键词: 海洋维权; 博亦; 海上维权技术; 《联合国海洋法公约》

中图分类号: D814.9, F224.32 文献标识码: A

\title{
Game Theory Analysis of Marine Rights Protection
}

\author{
Wang Lei ${ }^{1}$, Chen Hua ${ }^{2}$, Kang Meize ${ }^{3}$, Ma Xiaochuan ${ }^{1}$ \\ (1. Institute of Acoustics, Chinese Academy of Sciences, Beijing 100190, China ; 2. Business College of Beijing Union University, \\ Beijing 100025, China; 3. China Ship Development and Design Center, Wuhan 430064, China)
}

\begin{abstract}
This study discusses marine rights protection from the perspective of game theory. It introduces the role of marine rights protection in handling marine disputes, as well as the nature, direction, and elements of the game in China's marine rights protection. Currently, to minimize marine disputes through the game theory and pursue joint governance accord with China's national interest. To this end, this study categorizes marine rights protection into three basic game processes, i.e., entry to disputed sea areas, cross-border operation by fishing boats, and non-cooperative fishing, and then conducts corresponding game analysis to determine the key directions for building the marine right protection force of China. It is concluded that improving technological means for marine rights protection can effectively change the game revenue and achieve a beneficial game equilibrium. In particular, the development of technologies for high-tech interference, underwater protection, marine wide-area surveillance, and remote sea fishery development can assist China to gain an advantageous position in repeated game processes.
\end{abstract}

Keywords: marine rights protection; game theory; marine rights protection technology; United Nations Convention on the Law of the Sea

收稿日期 : 2019-06-21; 修回日期 : 2019-11-18

通讯作者: 陈华, 北京联合大学商务学院副教授, 主要研究方向为绩效管理; E-mail: hua.chen@buu.edu.cn

资助项目：中国工程院咨询项目 “海洋强国战略研究 2035” (2018-ZD-08)

本刊网址：www.engineering.org.cn/ch/journal/sscae 


\section{一、前言}

海洋是人类社会赖以存续的基础, 海洋资源的 开发水平及其健康状况直接影响人类社会的可持续 发展能力。《联合国海洋法公约》(UNCLOS) [1] 规 定了沿海国最多 $24 \mathrm{n}$ mile 毗连区和 $200 \mathrm{n}$ mile 的专 属经济区，赋予沿海国在专属经济区内的权利、管 辖权和义务, 使海洋具有了 “蓝色国土” 的意义。 进入 21 世纪, 我国在地缘政治上实现崛起, 在经 济上与全球产业链密切融合, 对海洋权益事关国家 安全和长远发展利益有了新的认识, 从而在维护海 洋权益方面更为积极主动。与此同时, 世界上部分 国家在零和博弯思维下对我国的快速发展产生了战 略焦虑, 导致与我国的海洋争端频发。典型事件有: 2012 年钓鱼岛 “购岛事件”，2013 年菲律宾发起 的 “南海仲裁案”，2014 年南海 “981” 钻井平台 冲突事件等。相关事件使得海洋争端迅速升级, 海 洋维权斗争形势异常复杂尖锐。这类维权斗争以参 与方的执法力量直接对抗为主, 维权博弯的范围涉 及政治、经贸、军事、外交等方面，处理不当则有 危机失控的风险。

作为海洋维权重要组成部分的海上维权, 处于 维权斗争的一线，具有高度的政治性和敏感性。一 般意义上, 海上维权是权益主体对其管辖海域范围 内发生的侵权活动，依据法定职权而实施管理的一 种行为 [2]。UNCLOS 第 73 条规定了沿海国在其专 属经济区可采用多种方式行使主权权利, 以及现行 海上维权通行的基本手段和方式，而依据 UNCLOS 进行维权执法的前提是执法海域的主权属性没有争 议。由于处理海洋主权争议通常更为复杂棘手，因 此一定阶段内, 我国在周边海洋争端中采取一定程 度上模糊主权属性的维权执法就成为常态; 不仅需 要对行使管辖权进行灵活把握, 还需要根据周边政 治、经济环境变化进行必要调整。在顶层设计上, 为了约束和管控海洋争端和海上维权斗争，我国与 东盟在《南海各方行为宣言》(DOC) 基础上, 达 成了 “南海行为准则”（COC）框架文件，基本建 立南海危机管控和沿岸国合作机制, 排除了域外 国家干扰，形成了积极成果。但同时应清晰看到， COC 确定后围绕渔权及配额分配、海洋资源勘探开 发等方面的激烈博弯将长期存在, 需要在海洋开发 能力、维权技术和应对策略方面预作准备, 做到进
退有据，以有效维护我国海洋权益。

国内在海洋维权问题研究方面开展了较多工 作。关于我国海洋争议与维权现状, 王红霞等 [3] 给出了我国东海、南海权益被侵犯的历史及现状; 李志文 [4] 详细分析了当前南海海洋争议及维权困 局。关于维权法律体系建设, 范金林等 [2] 指出我 国现有的海洋法律体系尚缺乏统一规划, 造成海洋 法律体现出边缘性和从属性。在维权执行层面, 丁 晓军等 [5] 认为争议海域维权应坚持 “国家主权利 益至上原则” “和平利用资源原则” “合作解决海洋 问题原则”。张永等 [6] 归纳了海洋维权的 5 类手段: 宣示手段、法律手段、执法力量手段、海洋开发手 段和多方合作手段。吴强等 [7] 针对外国军事测量 及非法测绘行为, 建议加强预防、随船与现场执法, 以及国内相关法律管辖下的责任追究等。

由于历史及地理因素, 我国与周边多个沿海国 存在着十分复杂的海洋争端, 海洋维权斗争形势呈 现出多样化特征, 在认识方面也有容易混淆之处。 同时应该注意到, 我国海洋维权任务以国家软、硬 实力为后盾，服务于海洋资源开发利用和 “海上丝 绸之路” 战略大局, 具有长期性、敏感性和突发性。 如何能把握维权斗争均衡状态, 使得海洋维权斗争 进退有据、海上维权执法规范有效, 这是当前海洋 维权力量建设中亟需研究的问题。因此, 本文对我 国海上维权的性质和特点进行分析，合理区分海洋 争端下国家维权斗争、一定协议约束下的维权执 法; 开展 “争议海区进入” “渔船越界维权” “非合 作渔业博亦模型” 应用研究, 分析海洋维权斗争的 能力需求; 在此基础上探讨维权科技的支撑作用及 价值。

\section{二、海洋维权的博栾问题}

\section{（一）海洋维权在处理海洋争端中的作用}

根据海洋争议涉及的利益和影响范围, 海洋争 端首选解决方式是通过当事国划界谈判予以消除, 如中越北部湾划界案。第二种解决方式是暂时搁置 争议, 并就海事、渔业等作出事务性安排, 如中韩 东海划界争议，大体上属于某种形式的联合管控。 这两种方式通过形成具有约束力的协议, 由当事国 建立某种合作机制, 相应的海上维权形式主要体现 为海上执法力量与侵权个体（如越界渔船）之间的 
博弯。第三种解决方式是海洋维权斗争, 即通过维 权方式实现争议海区宣示和管控, 例如 “钓鱼岛争 端”。当事国通过常态化巡航行动, 由执法力量形 成某种对抗均衡状态，达到主权宣示目的。第四种 解决方式是军事冲突或战争, 通过领土或岛屿占领 实现海区控制（见表 1)。

\section{（二）我国海洋维权博弯性质、方向的设定}

在宏观层面上, 海洋维权首先是国家对海洋开 发的理念和政策的博弯，避免因短期利益考量下的 海上冲突升级甚至国家对抗。中国政府提出 [8]: “中 国全面参与联合国框架内海洋治理机制和相关规则 制定与实施，落实海洋可持续发展目标” “实现海 洋资源有序开发利用”。一方面, 我国海洋维权的 性质在总体上不是对抗性的, 而是为了实现海洋资 源有序开发利用，这也包括合理分配; 另一方面， 我国海上维权斗争不仅要维护区域和平, 还需要全 面参与联合国框架内海洋治理机制和相关规则的制 定与实施（见表 2) [9]。

需要认识到, 当今的全球环境和海洋状况与 20 世纪 80 年代相比有了很大不同, 海洋健康状况 退化明显。过去 40 年中, 海洋中塑料污染增加了 10 倍, 脱氧死亡区的范围和数量都在持续增加, 海 洋酸化、海平面上升和气候变化的不利影响正在 造成巨大损失 [10]。根据联合国粮食及农业组织 (FAO) 估计 [11], 在生物学不可持续水平上的捕 捞鱼类种群比例从 2013 年的 $31.4 \%$ 上升到 2015 年
的 33.1\%, 鱼类种群状况恶化的长期趋势仍在延续。 近年来, 针对海洋污染防治, 生物多样性保护, 制 止过度、非法、未报告和无管制的捕捞活动及破坏 性捕捞做法等方面，国际社会正在凝聚共识并逐步 形成具有约束力的规则。这对我国的维权斗争既是 挑战, 更是机遇, 应准确把握维权斗争发展的方向 和进展，在维护好海上合作大局的前提下，推动海 洋维权博弯向微观化、事务性方向发展, 全面平衡 地维护好我国的海洋权益。

\section{（三）维权博栾模型的要素}

海洋维权行为作为低烈度非合作博弯问题, 从 维权斗争向联合管治方向发展符合我国国家利益。 一般而言, 海洋维权博弯模型较为复杂, 维权海区 范围和维权强度等也随着博弯双方力量对比、国际 形势变化而有所不同。

以南海争议为例, 维权博弯模型的基本要素 有: 博亦参与方（中国、南海六国、美国及南海地 缘关联国、其他国家), 侵权行为, 维权对策, 维 权收益等。侵权行为可细分为: 侵犯性海洋立法行 为, 争议海区油气勘探与开采、海事、渔业纠纷, 非法侦测、测绘及无人岛礁活动等。而非法岛礁占 领和 “自由航行” 名义下的军事巡航应归类于准军 事对抗行为, 不属于海上维权范围。维权对策可细 分为: 国内涉海立法, 领导人及政府宣示, 舰船/ 飞机巡航; 海事及渔业执法, 护渔行动; UNCLOS 规定的对侵权船只 “紧追、登临、检查、扣押和驱

表 1 海洋争端的解决方式

\begin{tabular}{llcc}
\hline 解决方式 & 海区范围 & 博亦方式 & 数学模型 \\
\hline 划界谈判 & 争议海区 & 非合作博亦 & 完全信息动态 bargaining 问题, 子博亦完美 (SPE) \\
联合管治 & 争议海区 & 合作博亦 & 所得与所付出的贡献相对应 (Shapley 指数 [12]) \\
维权斗争 & 争议海区 & 非合作博亦 & 长期参与方的重复博亦 \\
战争 & 全部海区 & 非合作博亦 & 能力、代价与承受力模型 \\
\hline
\end{tabular}

表 2 我国签署的海洋公约和协议情况（部分）

\begin{tabular}{|c|c|c|c|c|}
\hline 公约协议 & 签署日期 & 批准日期 & 缔约国家数 & 周边沿海国家签署情况 \\
\hline 《联合国海洋法公约》 & 1982 年 12 月 10 日 & 1996 年 6 月 7 日 & 168 & 日本、韩国、南海六国 \\
\hline $\begin{array}{l}\text { 关于执行《联合国海洋法公约》第 } \\
\text { 十一部分的协定 }\end{array}$ & 1994 年 7 月 29 日 & 1996 年 6 月 7 日 & 150 & 日本、韩国、南海六国 \\
\hline $\begin{array}{l}\text { 执行《联合国海洋法公约》有关养 } \\
\text { 护和管理跨界鱼类种群和高度洄游 } \\
\text { 鱼类种群的规定的协定 }\end{array}$ & 1996 年 11 月 6 日 & 未批准 & 90 & $\begin{array}{l}\text { 日本、韩国、菲律宾、越南、 } \\
\text { 泰国、印度尼西亚 }\end{array}$ \\
\hline
\end{tabular}


逐”; 设立海洋生态保护区; 海上对峙及冲突等。 维权博弯收益包括: 国家主权及声誉, 直接海洋经 济利益, 博弯双方的政治、经贸关系, 以及维权投 入等。

总体而言, 我国周边海域维权形势可控, 国际 政治环境在短期内不会有来自海上的直接威胁; 在 南海和东海方向, 与美国、日本等国处于一定均衡 态势; 南海六国与我国有重要经贸利益, 作为理性 参与者, 也倾向于维持一定阶段的博弯均衡状态。

\section{（四）海上维权博弯的基本模型}

海上维权博弯过程可由不同层次的博弯模型来 表征。在一定阶段, 一定维权内容的约束下, 可采 用简化的基本博弯模型来研究南海维权博弯过程。 作为典型案例, 本文分别讨论争议海区进入模型、 合作模式下的渔船越界模型、非合作模式下的渔业 维权模型。

\section{1. 争议海区进入模型}

20 世纪下半叶, 我国对争议海域的开发活动十 分谨慎, 使得周边国家以很小代价进行争议海区的 开发和侵权。目前在部分海区, 我国处于恢复主张 合法合理权益的一方, 需要考虑进入海区的博弯问 题。类似于市场进入，争议海区进入模型的博弯树 如图 1 所示, 其中的收益值均为假定值, 用于模型 均衡态分析。图 1 中的 $(-100,500)$ 表示, 维权 方选择进入海区后, 侵权方选择冲突时, 维权方收 益为 -100 , 而侵权方收益为 500 。

在海区进入博峦中, 存在两个纳什均衡: (1)维 权方进入海区进行开发, 侵权方威胁发生冲突, 维 权方选择不进入海区进行开发; (2)维权方进入海 区, 侵权方选择容纳, 在开发过程中双方逐步形成 某种合作机制。

本模型的典型案例之一是中国、越南围绕南海

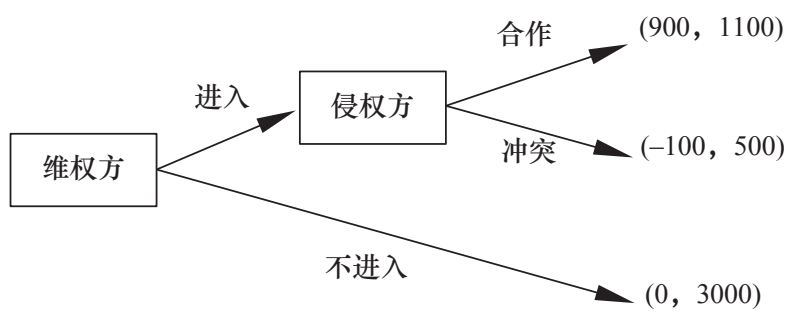

图 1 争议海区进入模型的博栾树示意图
“981” 钻井平台的冲突事件, 越方采用冲突选项进 行威胁, 试图达到阻止我方进入的纳什均衡。该事 件发展过程表明: 海洋维权属于重复博弯过程, 冲 突和干扰作为阻止进入的手段，一般被侵权方优先 使用; 在下一阶段对方进入争议海区时, 冲突和干 扰手段将同样被采用; 如果干扰手段效果更为明 显, 则进入方在冲突中损失将更大, 也就可以在更 长的时间周期、更大的争议海区范围内达到阻止进 入 (侵权) 的效果。此外, 我方进入争议海区进行 开发时, 应当做好防护防卫工作, 保证转入冲突模 式后的收益, 同时使对方冲突代价上升，不得不转 向合作开发的纳什均衡。

\section{2. 渔船越界维权博弯}

在当事国间建立了渔业协定的条件下，维权博 弯的对象便成为了越界渔船。由于维权力量的有限 性, 只能以一定概率处罚渔船越界捕鱼行为, 这归 属于博弯论中的混合策略博弯问题, 简单的博弯矩 阵见表 3 ( $p$ 为处罚概率, $q$ 为越界概率)。

对渔船而言, 被处罚的概率越高、处罚越严厉, 则其越界的意愿就越低。提高发现和处罚概率就成 为维权一方主要的工作方向。在合作博弯中, 越界 处罚程度受到一定的约束, 如表 3 中 $a$ 取值为 -1 。

令渔船越界和不越界捕鱼的预期收益相等, 即 $-p+5(1-p)=p+1-p$, 则 $p=2 / 3$ 。这说明, 受 执法方处罚的概率为 $2 / 3$ 时, 如果渔船没有风险偏 好, 渔船越界的概率 $q=0.5$ 。在该模型中, 渔船无 风险偏好的越界概率可以表示为:

$$
q= \begin{cases}-\frac{3}{2} p+\frac{3}{2}, & 1 / 3 \leqslant p \leqslant 1 \\ 1, & 0 \leqslant p<1 / 3\end{cases}
$$

在某指定海域存在 $N$ 条渔船条件下，未发生越 界侵权的概率为 $(1-q)^{N}=\left(\frac{3}{2} p-\frac{1}{2}\right)^{N}$ 。假定某海区 存在 10 条潜在越界渔船, 而处罚概率为 0.9 , 可以 计算出不发生越界捕捞的概率仅为 0.2 。由此可见,

\section{表 3 渔船越界维权博弯矩阵}

\begin{tabular}{lccc}
\hline & $\begin{array}{c}\text { 维权执法方 } \\
\text { 处罚 }\end{array}$ & $\begin{array}{c}\text { 维权执法方 } \\
\text { 未处罚 }\end{array}$ & 越界概率 \\
\hline 渔船越界 & $(a, 2)$ & $(5,-1)$ & $q$ \\
渔船不越界 & $(1,0)$ & $(1,0)$ & $1-q$ \\
处罚概率 & $p$ & $1-p$ & \\
\hline
\end{tabular}


在 0.9 的处罚概率下, 仍将有 0.8 的概率出现越界 捕捞, 可见维权监视的任务是十分繁重的。

\section{3. 非合作渔业博弯模型}

南海传统渔业资源主要分布在陆架和岛礁区 域, 有一定的渔获周期, 开展海洋牧场建设可以扩 展传统的渔获区域范围。为了简化问题研究, 设定 在某一渔获期间, 没有外部影响 (如双方主权宣示 要求), 双方护渔行为设定为驱赶对方渔船。假定 争议海区面积为 $\mathrm{S}$, 渔民为风险厌恶型, 仅在各自 护渔范围作业; 假设单位面积渔获收益相等, 博弯 双方渔业捕捞技术水平和成本存在一定差异。

综合考虑渔获收益和维权代价, 单位 (面积) 综合收益 $p$ 是护渔巡航面积 $s$ 的递减函数 (图 2 中 以实线表示), 其属性是由地理位置远近以及远海 捕鱼能力所决定的。为了得到最佳决策点 (收益最 佳), 可以绘制一组等收益曲线 $s p=C$ 。随着总收 益 $C$ 的变化, 等收益曲线会与单位收益曲线相切, 切点即为最佳决策点 (图 2 中的 $A$ 点)。当捕捞效 率较高时, 护渔单位收益将增加, 收益函数下降也 会变缓, 最佳决策点 (综合收益最大) 将向更大面 积方向移动 (见图 3 中 $B$ 点)。

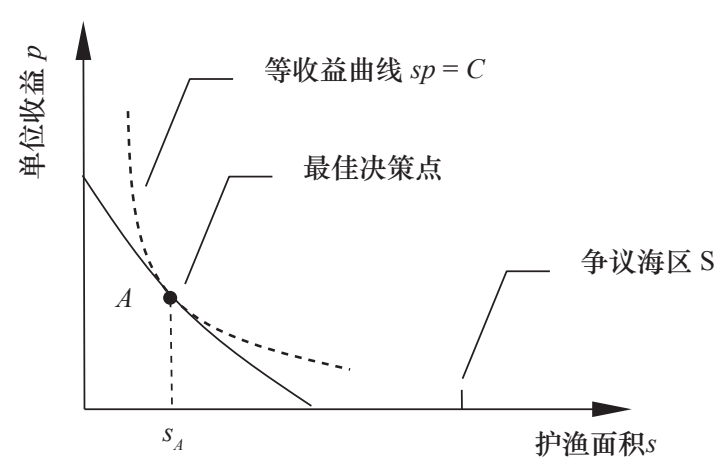

图 2 非合作渔业博弯的综合收益曲线

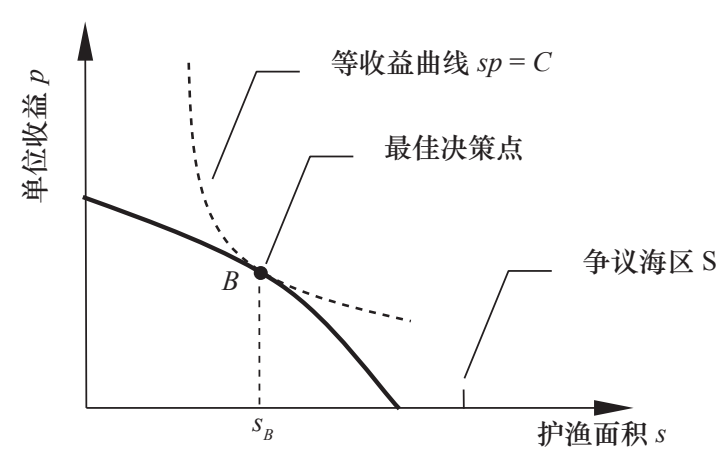

图 3 高效率方的综合收益曲线
在具体执法过程中, 护渔面积 $s(0 \leqslant s \leqslant \mathrm{~S})$ 作为决策变量, 可以选择争议海区巡航范围达到靠 近对方一侧, 也可选择在靠近己方一侧巡航。如果 双方护渔面积发生重叠, 就会引发冲突, 双方都会 产生额外损失。将双方的综合收益曲线绘制在同一 张图上 (见图 4), $s_{\mathrm{A}}$ 为护渔方 $\mathrm{A}$ 的最佳决策点的 护渔区域位置, $s_{\mathrm{B}}$ 为护渔方 $\mathrm{B}$ 的最佳决策点的护渔 位置。当 $s_{\mathrm{A}}>S_{\mathrm{B}}$ 时, 双方将发生护渔冲突, 并分别 受到损失 $L_{1}$ 和 $L_{2}$, 均衡点将移动到 $s_{1}, s_{2}$ 之间。找 到均衡点后, 可快速实现纳什均衡, 提高维权效率。 在实际工作中, 双方维权人员会依据经验寻找和评 估均衡点位置。

从图 4 来看, 护渔的均衡点是以双方远海捕捞 能力为基础的, 远海渔业能力差的一方将面临不利 的均衡状态, 这与我国南海渔业现状相符。当前南 海近海区域处于过度捕捞状态, 渔业资源枯竭, 亟 需养护; 南沙、中沙及远海相当部分区域处于欠捕 捞状态 $[13,14]$ 。由于我国在南海远海渔业捕捞方面 能力不足, 与越南、菲律宾相比技术落后 (日本通 过东南亚渔业开发中心 SEAFDEC 等途径长期提供 技术支持), 经济性差; 我方的远海维权执法能力 难以体现为具体收益, 在未来渔业合作和捕捞配额 分配方面, 远海渔获能力不足的短板将更为突出。 因此关于这一博弯问题, 提高综合收益将是关键性 举措, 在图 2 图 4 上表现为收益曲线向远海 (更 大面积）延伸。

建议快速发展远海渔业, 与我国目前远海维 权能力相适配。可采取的实际措施包括: 鼓励成 立较大规模远海渔业捕捞公司, 吸纳近海捕捞作 业渔民, 发展远海渔业装备, 提高捕获效率; 建 立远海渔业加工、服务等配套产业; 发展远海牧

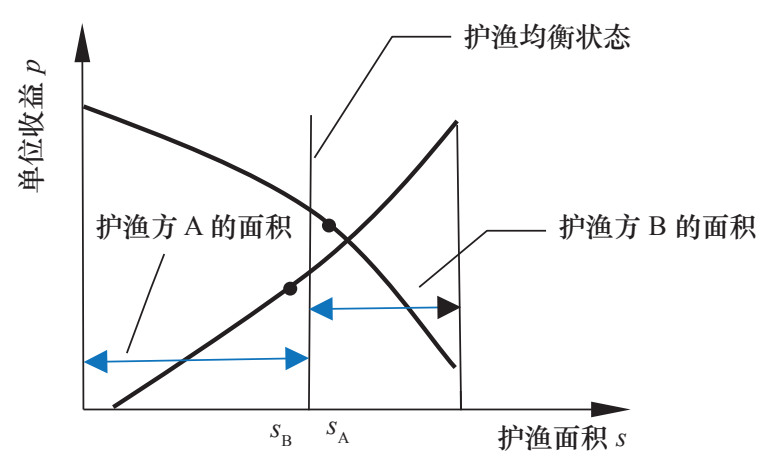

图 4 护渔维权的纳什均衡示意图 
场，扩大远海渔业产业等。

\section{三、增强海上维权博栾能力的途径}

根据非合作渔业博栾模型可见，海洋维权的基 础在于海洋产业发展的能力和水平。远海渔业, 特 别是洄游性高价值鱼类 (如金枪鱼) 捕捞, 需要渔 用声纳、高端渔机等探渔助渔装备。由于在渔业科 技方面历史欠账多、重视程度低, 我国先进渔用声 纳国产率为零, 相关市场被挪威 SIMRAD、日本谷 野等公司占据。因此, 函需发展海洋产业科技, 夯 实海洋维权的装备和能力基础。

根据海区进入模型可见, 为了进入争议海区, 需要解决冲突中的防卫问题。水下空间防卫成为重 点, 如在南海 “981” 钻井平台冲突过程中, 越南 方面派出蛙人进行威胁。需要发展对水下目标的侦 测和应急处理能力, 在海区进入冲突状态时保障和 提高我方收益。例如, 在海南省海洋牧场建设规划 中, 计划在中沙和南沙附近建立 10 个远海海洋牧 场, 除了警示浮标外, 还需建立水下网箱监控防护 系统, 以防范专业蛙人破坏水下网箱导致鱼苗流失 的风险。为了阻止侵权方进入争议海域作业, 如油 气勘探、海上测绘等活动, 需要发展高技术冲突干 扰手段, 如远程智能声学压制, 假目标欺骗、水下 无人潜航器 (UUV) 抵近干扰等手段, 大幅降低侵 入方在冲突中的收益, 从而阻止对方侵入。

根据越界渔船维权模型可见, 发展低成本高效 率的维权监视系统极为必要。例如, 建立基于渔船 联网的水面监视取证系统、空中无人机巡航作业系 统等, 可显著提高对越界渔船的处罚概率。

发展海洋产业与科技、提高海上维权能力, 可 以有效改变博弯收益, 进而影响博弯决策, 获取有 利我方的博弯均衡。依靠科技进步可以有效提高我 方维权执法效率和水平, 降低维权冲突代价, 大幅 提高国际舆论对我方维护海洋权益的认可度。维权 科技发展与海洋大学科的整体前瞻规划密不可分, 这也包括了深远海等维权重点区域。例如：(1)海上 燃油泄漏及污染物扩散问题与海洋气象及洋流活动 关系密切, 我国应当尽快建立深远海气象水文、洋 流活动监测和数值预报系统; (2)作为海上维权的重 要内容, 海洋生物多样性及生态环境保护方面近年 来日益受到重视, 目前国际上已对 $18 \%$ 的辖区内
海域和 $1 \%$ 的公海区域设定保护区 [11], 我国需要 加强海洋生态学研究, 以进一步规范远海渔业捕 捞, 为生态物种多样性保护提供依据, 进而提高 国家声誉。

\section{四、政策与建议}

（1）海洋维权的基础是海洋开发的能力和水平, 建议加快海洋新兴产业技术的发展, 做到海洋环境 保护和资源有序开发上的协同, 打牢下一阶段海洋 合作博亦的基础。

（2）针对海上维权的能力建设要求, 建议开发 海上维权新技术手段, 包括研制应用于冲突干扰和 防卫防护的新技术装备, 改变双方博亦收益, 实现 有利于海上维权的纳什均衡。

（3）海上维权博弯本质上高度综合复杂, 建议 加强产业、自然资源、科技、信息传媒、外交及海 警等方面的政策协调，形成联动机制以综合应对突 发事件。

（4）准确把握海上合作和维权的大局, 建议在 海洋环境治理、生态资源保护和海洋资源有序开发 方面着力, 建立自主可控的深远海观测系统, 形成 海洋水文及动力学环境、大型鲸类和海上船舶动态 监控能力。在海上灾害管理和应急处置方面, 建立 快速干预系统, 发展可靠的深远海精确定位及远程 指挥控制能力, 开展海洋信息科技建设, 将海上维 权博弯向高质量方向推进。

（5）在海上维权实践的基础上，建议对我国现 有维权力量进行优化整合, 配置或研发多样性的维 权技术手段，提高维权效率。建立维权对策专业研 究机构, 深入海上维权一线, 解决日常维权中的突 出矛盾，对突发事件进行战略预判并做好应急策略 部署。

\section{参考文献}

[1] 联合国. 国际海洋法公约 [EB/OL]. (1982-12-10) [2019-05-12]. https://www.un.org/Depts/los/convention_agreements/texts/unclos/unclos_c.pdf.

United Nations. United Nations convention on the law of the sea [EB/OL]. (1982-12-10) [2019-05-12]. https://www.un.org/Depts/ los/convention_agreements/texts/unclos/unclos_c.pdf.

[2] 范金林, 郑志华. 重塑我国海洋法律体系的理论反思 [J]. 上海 行政学院学报, 2017, 18(3): 105-111. 
Fan J L, Zheng Z H. Reflections on the reconstruction of China's marine legal system $[\mathrm{J}]$. The Journal of Shanghai Administration Institute, 2017, 18(3): 105-111.

[3] 王红霞, 李真龙, 高婷婷. 我国东海和南海海洋维权的现状和展 望 [J]. 法制与社会, 2015 (4): 167-168.

Wang H X, Li Z L, Gao T T. The present situation and prospect of China's maritime rights protection in the East and the South China Sea [J]. Legal System and Socity, 2015 (4): 167-168.

[4] 李志文. 我国在南海争议区域内海上维权执法探析 [J]. 政法论 从, 2015 (3): 92-99.

Li Z W. On the law enforcement of the disputed area in South China Sea [J]. Journal of Political Science and Law, 2015 (3): 92-99.

[5] 丁晓军, 王婧. 南海争议海域维权困境及路径 [J]. 中国青年社 会科学, 2017, 36(4): 124-130.

Ding X J, Wang J. The dilemma and path of safeguarding rights in the disputed waters of the South China Sea [J]. Journal of China Youth University for Political Sciences, 2017, 36(4): 124-130.

[6] 张永, 姚建新. 海洋维权手段探要 [J]. 海洋开发与管理, 2008, 25(3): 76-79.

Zhang Y, Yao J X. Explore the means of safeguarding maritime rights [J]. Ocean Development and Management, 2008, 25(3): 76-79.

[7] 吴强, 赵胜汝. 海洋权益维护执法对策分析 [J]. 海洋开发与管 理, 2004, 21(6): 38-43.

Wu Q, Zhao S R. Analysis on the maintenance and enforcement of the law for ocean rights and interests [J]. Ocean Development and Management, 2004, 21(6): 38-43.

[8] 新华网. 习主席海洋命运共同体理念引共鸣 [EB/OL]. (2019-0608) [2019-06-08]. http://www.xinhuanet.com//world/2019-06/08/ c_1210153933.htm.
Xinhuanet. President Xi's vision of a maritime community of shared future resonates [EB/OL]. (2019-06-08) [2019-06-08]. http://www.xinhuanet.com//world/2019-06/08/c_1210153933.htm.

[9] United Nations. Current status of the convention, table of ratifications/accessions [EB/OL]. (2019-08-31) [2019-08-31]. https:// www.un.org/Depts/los/reference_files/status2019.pdf.

[10] United Nations. 海洋和海洋法——秘书长的报告 [EB/OL]. (2019-09-11) [2019-09-11]. https://undocs.org/zh/A/74/350.

United Nations. Oceans and the law of the sea-Report of the secretary-General [EB/OL]. (2019-09-11) [2019-09-11]. https:// undocs.org/zh/A/74/350.

[11] United Nations. 海洋和海洋法——秘书长的报告 [EB/OL]. (2018-09-05) [2018-09-05]. https://undocs.org/zh/A/73/368.

United Nations. Oceans and the law of the sea-Report of the secretary-General [EB/OL]. (2018-09-05) [2018-09-05]. https:// undocs.org/zh/A/73/368.

[12] Drew F, Jean T. 博亦论 [M]. 黄涛 译. 北京: 中国人民大学出版 社, 2010.

Drew F, Jean T. Game Theory [M]. Translated by Huang T. Beijing: China Renmin University Press, 2010.

[13] 张俊, 邱永松, 陈作志, 等. 南海外海大洋性渔业资源调查评估 进展 [J]. 南方水产科学, 2018, 14(6): 118-127.

Zhang J, Qiu Y S, Chen Z Z, et al. Advances in pelagic fishery resources survey and assessment in open South China Sea [J]. South China Fisheries Science, 2018, 14(6): 118-127.

[14] 李斌, 陈国宝, 郭禹, 等. 南海中部海域渔业资源时空分布和资 源量的水声学评估 [J]. 南方水产科学, 2016, 12(4): 28-37.

Li B, Chen G B, Guo Y, et al. Hydroacoustic assessment of spatialtemporal distribution and biomass of fishery resources in the central South China Sea [J]. South China Fisheries Science, 2016, 12(4): $28-37$. 